\title{
Cancer cell aggregate hypoxia visualized in vitro via biocompatible fiber sensors
}

\author{
Ruipeng Xue ${ }^{1}$, M. Tyler Nelson ${ }^{3}$, Silvia A. Teixeira ${ }^{2}$, Mariano S. Viapiano ${ }^{2}$, John J. Lannutti ${ }^{1,3}$
}

1. Department of Materials Science and Engineering, The Ohio State University, Columbus $\mathrm{OH}$

2. Department of Neurosurgery, Brigham and Women's Hospital and Harvard Medical School, Boston MA

3. Department of Biomedical Engineering, The Ohio State University, Columbus, $\mathrm{OH}$

\section{Abstract}

To fully understand biological behavior in vitro often dictates that oxygen be reported at either a local or a cellular level. Oxygen sensors based on the luminescent quenching of a specific form of electrospun fiber were developed for measurement of both gaseous and dissolved oxygen concentrations. Electrospinning was used to fabricate "core-shell" fiber configurations in which oxygen-sensitive transition-metal porphyrin complexes are embedded in an optically clear, gas permeable polycarbonate polymer 'core' while polycaprolactone provided a protective yet biocompatible 'shell.' By taking advantage of the resulting high sensitivity and fast response of electrospun core-shell fiber sensors, we were able to locate and image hypoxic regions in contact with aggregates of glioblastoma cells. Nanoscale, biomimetic sensors containing oxygen-sensitive porphyrins are particularly well suited to biological applications. These 'smart' nanofiber based sensors do not consume oxygen, their mechanical 
and chemical characteristics can be finely tuned allowing tailoring of biocompatibility and microstructure. Core-shell nanofiber oxygen sensing fibers could provide real-time assessments of tumor cell response to pharmacological innovations designed to target hypoxic regions driving new knowledge and technological advancement.

Keywords: hypoxia, porphyrins, core-shell, tumors, polycaprolactone, polycarbonate 
Keywords:

A common feature of advanced solid tumors is the presence of hypoxic regions distributed heterogeneously throughout the overall tumor mass. [1] Hypoxic regions arise from the imbalance between the increased cell mass utilizing oxygen and a deficient supply caused by structural and functional tumor blood vessel abnormalities, increased diffusion distances and tumor-associated or therapy-induced anemia. [2] Hypoxia is associated with resistance to chemotherapy because it limits the availability of molecular $\mathrm{O}_{2}$ to contribute to cytotoxicity through the deliberate generation of free radicals. [3] It also indirectly influences the effectiveness of radiation therapy by inducing proteomic and genomic changes that increase tumor invasiveness and metastatic potential. [4] In particular, hypoxia plays an important role in recruiting stromal cells that change the composition and structure of the extracellular matrix (ECM) [5] and remodel the tumor to potentiate malignant cell proliferation and dispersion. As a result, patients with poorly oxygenated primary tumors $\left(P_{\mathrm{O} 2}<10 \mathrm{~mm} \mathrm{Hg}\right)$ demonstrate a higher risk of metastasis and mortality. [6] Given that new pharmacological compounds targeting these tumors are initially tested in vitro, rapid local characterization of gradients in oxygen around cultured cell agglomerates is desired to better understand and probe the inherent physiology of hypoxic cells.

To advance chemotherapeutic development, techniques allowing localized measurements of molecular oxygen in the tumor microenvironment - either in vitro or in vivo are of great interest. Appropriate sensors should be easy to use, readily integrated with standard cell/tissue culture devices and microscopes, and exhibit suitable biocompatibility. One of the dominant oxygen sensors for biological applications is the Clark electrode. The Clark electrode benefits from an inherent reliability and lack of heavy metal interference potentially preventing oxygen ion diffusion through the sensing membrane. However, Clark electrodes are invasive and consume oxygen during measurement. They are unequipped to 
provide oxygen distributions for biological applications due to their bulky size and configuration that carries with it the potential for contamination. [7]

Optical methods based on dynamic quenching of luminescence are attractive and promising in biologically related applications due to the following: high sensitivity and accuracy, non-invasive measurement, easy signal readout or oxygen distribution imaging, lack of oxygen consumption and potential for miniaturization down to the scale of individual cells. [8, 9] The most frequently utilized molecular probes consist of transition metal-ligand complexes known as metalloporphyrins. These compounds are typically dissolved in a protective matrix because they might otherwise have limited oxygen accessibility, suffer from self-quenching and can potentially be cytotoxic. Fortunately, these same probes generally exhibit better luminescence and photostability when fully dissolved in such solid matrices.

Although most reported optical oxygen sensors are film based, sensors can take many other forms depending on the requirements of the specific application. Polyurethane hydrogelcontaining palladium porphyrin doped polystyrene-co-acrylonitrile particles were used as 2D luminescence sensing film for imaging dynamic physiological wound oxygenation. [10] Nanosized sensing particles can allow for intracellular oxygen imaging. [11] In closely related tissue engineering applications, oxygen diffusion was studied within collagen-based tissue constructs using an optical fiber-based oxygen sensor. [12] Compared to these techniques, electrospun fiber based sensors have considerable potential for productive in vitro studies due to the fact that the fiber form mimics both the topography and scale of the ECM while providing an oxygen reporting function "built-in" and [13, 14] is effective at the microscale [9].

In this work, malignant glioblastoma multiforme (GBM) cells were chosen as our biological model system, as GBM's are the most common tumors originating within the central nervous system (CNS) and account for over 15,000 deaths annually in the US [15]. The median survival for glioblastoma, the most common and aggressive of these tumors, is only 14 months despite aggressive surgical resection followed by adjuvant therapy [16]. A hallmark of 
glioblastomas is their highly invasive character exacerbated by exposure to hypoxic conditions $[17,18]$. The development of effective anti-invasive approaches for malignant gliomas has been largely hampered by the difficulty in modeling glioma cell migration appropriately in vitro [19]. We have previously developed a physiologically relevant model of glioma cell migration using ECM-mimicking electrospun polycaprolactone fibers that revealed dramatic differences in cell signaling and migration when tumor cells where challenged with different topographies (i.e., aligned versus random fibers) [20, 21]. This chemically and physically flexible model recapitulated in vivo cell morphology and allowed analysis of physiological mediators and pharmacological inhibitors of glioma cell migration [21], offering an excellent starting point for further optimization by including biosensors embedded in these fibers serving as a substrate for adherent cell culture.

In this study, new fiber-based oxygen sensors were developed and inoculated with glioblastoma cell aggregates in vitro to investigate the suitability of these sensors in studying tumor hypoxia. Electrospinning is a low-cost, versatile, single-step manufacturing technique extensively used in biological applications such as tissue engineering in which fibrous scaffolds serve as a matrix in which cells can attach, grow and differentiate. [22] Here, the fibers were fabricated by co-axial electrospinning [23] and consist of the biocompatible polycaprolactone $(\mathrm{PCL})$ shell and a highly stable polycarbonate $(\mathrm{PC})$ core containing an oxygen sensitive luminescence probe, $\mathrm{Pd}(\mathrm{II})$ meso-tetra(pentafluorophenyl) porphine (PdTFPP). Polycarbonate was selected as the matrix for this highly sensitive indicator due to its optical clarity, appropriate gas permeability, and mechanical stability. Taking advantage of the high sensitivity and fast response of these sensors, we are able to locate and image hypoxic regions beneath cultured GBM aggregates showing how these are preserved in spite of the presence of surrounding areas of normoxia. The widely utilized electrospinning platform provides opportunities to construct ECM-like structures having both controlled morphology and 
chemistry and integrated oxygen sensing enabling a better understanding of how regions of stable hypoxia can develop, be retained and subsequently control tumor cell behavior.

\section{Materials and methods}

\section{Materials}

Pd(II) meso-Tetra(pentafluorophenyl)porphine (PdTFPP) was obtained from Frontier Scientific Inc. Polycarbonate (PC) was acquired from Kotec Corporation and polycaprolactone (PCL) was from Sigma-Aldrich. Dichloromethane (DCM) was purchased from Sigma-Aldrich and 1,1,1,3,3,3-hexafluoro-2-propanol (HFP) was obtained from Oakwood Products Inc.

\section{Fabrication of core-shell fibers}

$2.5 \mathrm{mg}$ of luminescence probe PdTFPP was dissolved in a mixture of $2 \mathrm{~g} \mathrm{DCM}$ and $2 \mathrm{~g}$ HFP. $1 \mathrm{~g}$ of PC was then added to the solution that was then stirred at room temperature until a uniform solution was formed. PCL solution was prepared by simply dissolving 5 wt\% PCL pellets in HFP. Both solutions were loaded into separate $20 \mathrm{~mL}$ syringes connected to a coaxial flow device consisting of a $1.6 \mathrm{~mm}$ diameter compression tube fitting and a 16 gauge hypodermic tube fitting (both from Parker Hannifin, Cleveland, Ohio). Each syringe dispersed the solutions via pressure supplied by a programmed syringe pump. The PC solution was fed through the inner core of the device at a flow rate of $1 \mathrm{~mL} / \mathrm{h}$ while the $\mathrm{PCL}$ solution was fed at a rate of $4 \mathrm{~mL} / \mathrm{h}$ through the outer tube. A $25 \mathrm{kV}$ voltage was then applied between the coaxial needle and a grounded collector. Aluminum foil or $30 \mathrm{~mm}$ round glass cover slips were placed on the collector to obtain the resulting core-shell fibers for characterization or subsequent cell experiments. To better mimic the fibrillar nature of ECM scaffolds, fibers were fabricated in a similar fashion but were collected on a rotating metal wheel (25 inches in diameter, $500 \mathrm{rpm}$ ) to form fiber arrays displaying a general degree of alignment. All electrospun samples were placed in vacuum at room temperature overnight to completely 
remove possible solvent residue. [24] Fibers were also treated by a plasma cleaner (Harrick Plasma, Ithaca, New York) prior to cell culture to facilitate improved cell adhesion.

\section{Sensor calibration}

Initially, core-shell fiber mats were cut into $1 \times 1 \mathrm{~cm}$ squares glued to the inner wall of a glass cuvette. A cap having both a gas inlet and outlet was attached to top of the cuvette. The gas inlet was connected to a gas mixer (Omega Engineering, USA) that controlled the desired gas concentrations by adjusting the relative flow rate of nitrogen and oxygen. The blue LED light $(470 \mathrm{~nm})$ output from a fluorescence spectrometer (JAZ, Ocean Optics Inc., Dunedin, FL, USA) probe was perpendicularly directed at the fiber sample from the outside of the cuvette. The probe also contained a Si photodiode detector to collect emissions and transmit them to the spectrophotometer. To provide for calibration of dissolved oxygen levels, samples were immersed in water and specific oxygen concentrations continuously bubbled into the water. The actually dissolved oxygen (DO) level was verified by a commercial meter (HQ40d, Hach Company, Loveland, Colorado, USA). The calibration for both the gaseous phase and dissolved oxygen can be described by the Stern-Volmer equation

$$
I_{0} / I=1+K_{S V}\left[O_{2}\right]
$$

where $I_{0}$ and $\mathrm{I}$ are the luminescence intensity without any oxygen and with certain concentration of oxygen, respectively, and $\left[\mathrm{O}_{2}\right]$ is the corresponding oxygen concentration, either in volume percentage or in $\mathrm{mg} / \mathrm{L}$ for the case of dissolved oxygen. $\mathrm{K}_{\mathrm{sv}}$ is the SternVolmer coefficient, a direct measure of sensor sensitivity.

\section{Cell culture and aggregate production}

All cell cultures supplies were purchased from Fisher Scientific, Inc. unless otherwise stated. U251-MG (U251) human glioblastoma cells were purchased from ATCC. Tumor cells were cultured in DMEM/F12 medium containing $10 \%$ fetal bovine serum (FBS), $1 \%$ 
penicillin/streptomycin (P/S), and 1:500 MycoZap at $37^{\circ} \mathrm{C}$ and atmospheric conditions of $5 \%$ $\mathrm{CO}_{2} / 21 \% \mathrm{O}_{2}$. Cell Tracker green (Invitrogen, Inc, final dilution $1 / 10,000$ ) was added to U251 cells in suspension and incubated for 45 minutes to label cells for fluorescence microscopy. Aggregates of U251 cells were produced by inoculating 15,000 cells $/ \mathrm{cm}^{2}$ on round-bottom polystyrene 96 -well plate wells coated with a $1 \%$ hard-agarose gel layer. Cells aggregated in wells proliferated for 3 days to form $500 \mu \mathrm{m}$-diameter aggregates. Monodisperse U251 cells $\left(10,000\right.$ cells $\left./ \mathrm{cm}^{2}\right)$ and cell aggregates were seeded onto oxygen-sensing core-shell nanofibers and allowed to adhere for $12 \mathrm{~h}$ before conducting experiments. Normoxia experiments were conducted at $5 \% \mathrm{CO}_{2}: 21 \% \mathrm{O}_{2}$, while hypoxia was conducted at $5 \% \mathrm{CO}_{2}: 1 \%$ $\mathrm{O}_{2}$, balanced with $\mathrm{N}_{2}$. The cell aggregates remained on the fibers under either normoxic or hypoxic oxygen conditions for $48 \mathrm{~h}$ prior to microscopy or collection for downstream biochemical analysis.

\section{Biochemistry}

Dispersed cells cultured under normoxia or hypoxia were collected after $48 \mathrm{~h}$ and processed for SDS-PAGE and Western blotting following standard procedures to detect the

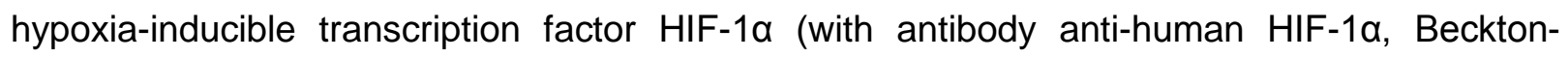
Dickinson Biosciences). Alternatively, hypoxic response was validated after $72 \mathrm{~h}$ in culture by measuring production of VEGF protein, a pro-angiogenic factor tightly regulated by HIF-1a. Briefly, conditioned medium from the cultured cells was used to detect secreted VEGF protein using conventional ELISA. Cells were also collected and RNA was extracted with Trizol reagent (Invitrogen) to measure expression of VEGF mRNA by semi-quantitative RT-PCR.

To determine if hypoxic exposure had affected the expression of the components involved in mitochondrial oxygen reduction (oxidative phosphorylation cascade, OXPHOS), cells were processed for Western blotting with an antibody cocktail to detect the expression of major OXPHOS proteins (Total OXPHOS antibody cocktail ab110411, Abcam). 
Imaging procedures and analysis

Fiber coated coverslips containing multiple cell aggregates were integrated to an interchangeable coverslip chamber (Bioptechs Inc., Butler, PA). The chamber was then placed in an incubator attached to the microscope stage with a heating lid to maintain the temperature at $37^{\circ} \mathrm{C}$. Controlled gas mixtures were passed through water at $37^{\circ} \mathrm{C}$ before introduction into the incubator. $5 \% \mathrm{CO}_{2}$ was maintained for all cell imaging experiments while oxygen concentration was varied to maintain either normoxia $(21 \%)$ or hypoxia $(1 \%)$. The total time required for the system to equilibrate at the desired oxygen concentration was $\sim 10$ minutes. $\mathrm{A}$ Nikon Eclipse TiE total internal reflection fluorescence (TIRF) microscope was used to image the fibers and cells using laser excitation at $435 \mathrm{~nm}$ (cells) and $561 \mathrm{~nm}$ (fibers).

Images were analyzed using ImageJ with an ND Viewer plugin to open the direct images from the microscope. Briefly, RGB images were separated into monochrome channels and the aggregates delineated manually to define "aggregate" and "periphery" (areas outside of the aggregate) regions in the image. The distribution of pixel intensities in the fibers was compiled for under-aggregate and periphery areas after subtracting background (black) pixels. The distribution of pixel intensities in the fibers was compiled for under-aggregate and periphery areas after subtracting background pixels defined as the lowest $15 \%$ of the pixel value distribution for each figure. A ratio of median pixel intensity for aggregate versus periphery (A/P ratio) was calculated to determine if the fibers in contact with tumor aggregates exhibited similar or different fluorescence intensity compared to fibers outside the aggregates.

\section{Results}

Sensor Performance

Co-axial electrospinning yielded core-shell fibers having a PdTFPP-containing polycarbonate core and an indicator-free polycaprolactone shell. Representative morphology 
of such fibers is revealed by SEM (Figure 1). The resulting fiber scaffolds possess small diameters, large surface areas and high porosity favoring cell proliferation, nutrient transfer and oxygen transport. Diameters of most fibers are in the range of 0.8-1.2 $\mu \mathrm{m}$ as shown in the histogram of fiber size distribution (insert of Figure 1). Upon excitation, fibers emitted red luminescence at $673 \mathrm{~nm}$ and its intensity was a function of the oxygen content of its surroundings. The observed emission is actually a phosphorescence involving transitions between a triplet excited state and a singlet ground state. The indicator has good quantum yield and clear separation (Stoke's shift) between the excitation and the emission peaks. These properties facilitate easy detection and measurement of the luminescence signals. As the emission spectra in Figure 1 shows, presence of increasing amounts of gaseous oxygen $(0-20 \%$ in volume $)$ significantly quenched the emission from the fiber core. The fundamental mechanism involves dynamic collision of the excited states of the luminescence probes with molecular oxygen. [8] The ultimate sensitivity $I_{0} / I_{100}$ value, defined as the intensity ratio of a sensor in oxygen-free environment $\left(I_{0}\right)$ versus pure gaseous oxygen $\left(I_{100}\right)$, was 131.6 when cycled between nitrogen and oxygen. For biological environments, the ideal sensors should be both sensitive to and selective for oxygen. They should be able to provide real time information relatively inexpensively. The high oxygen sensitivity of these fibers can also be visualized by fluorescence microscopy images taken at different oxygen concentrations (Figure 1). Polycarbonate is widely utilized in optical lenses due to its excellent transmissive properties and high impact resistance. However, it is not commonly used in oxygen sensors due to its low gas permeability $\left(96-101 \mathrm{~cm}^{3} \mathrm{~mm} / \mathrm{m}^{2}\right.$ day atm). Here, we show that $P C$ in electrospun fiber form can efficiently disperse the PdTFPP probe molecules, prevent interference by molecular species in biological environments while simultaneously allowing rapid responses to changes in oxygen tension.

Under rapid oxygen and nitrogen gas alternation, intensity peak was monitored continuously to reveal the reversibility and response time of the sensor. The response or 
recovery time $\left(t_{95}\right)$ is defined as time needed for $95 \%$ of the intensity change to take place when the environment is changed. The average response time from nitrogen to oxygen was $0.42 \mathrm{~s}\left(\mathrm{t}_{95 \downarrow}\right)$ and the recovery took a slightly longer time of $0.74 \mathrm{~s}\left(\mathrm{t}_{95 \uparrow}\right)$. The emission was not only fully reversible but also photostable during the entire reversibility test and extended stability test (data not shown). The fluorinated substituents of the porphyrin ring greatly increased the photostability of the complex. [25] Fiber mats were immersed in $37^{\circ} \mathrm{C}$ water with controlled dissolved oxygen concentrations to calibrate the response according to the SternVolmer equation. Sensors exhibited nearly perfect linear response (adj. $\left.R^{2}=0.9990\right)$ in the full biologically relevant range (Figure 2) reflecting the full range between absolute hypoxia and normoxia. A linear sensor response is highly desirable to enable ease of calibration. However, the majority of proposed optical oxygen sensors, unfortunately, exhibit a nonlinear SternVolmer response. This deviation from linearity makes calibration difficult and constitutes a major challenge for applying new sensor systems in biological situations. The origin of the experimentally observed nonlinear behavior is the non-uniform distribution of sensing probes or heterogeneous sensing environment. [26] Since the intensity dropped exponentially as dissolved oxygen was steadily increased, the largest intensity change happened in the range 0-2 mg/L. The intensity decreases exponentially as the dissolved oxygen gradually increased. When this intensity data is inserted into the Stern-Volmer relationship, the response becomes linear. Thus, these fibers are more sensitive under hypoxic conditions than in near airsaturated water. Pd porphyrins tend to have much longer lifetimes (400-1000 $\mu$ s) than its counterparts such as Pt porphyrins and could be used in environments with very low oxygen concentration (below $50 \mu \mathrm{M}$ ). [27] However, extreme sensitivity to even traces of oxygen does not suit applications involving large variations in oxygen so, in this context, the moderate oxygen permeable polycarbonate is then an appropriate choice of matrix which can actually limit the oxygen available to the Pd porphyrin molecules. 
For advanced applications, high spatial resolution and noninvasive measurement are highly desirable. The commonly seen macroscopic quenching coefficient is the averaged value from individual molecules and is the measurement of the average oxygen concentration around the bulk materials. Figure 3 shows the fluorescence images of a PC-PCL fiber exposed to different oxygen concentrations and imaged with a TIRF microscope. In the absence of oxygen, the fiber emitted a strong and steady red signal from the core when excited by $561 \mathrm{~nm}$ laser irradiation, a result of both high quantum yield of the porphyrin and good indicator-matrix compatibility. In $20 \%$ oxygen the fiber remained visible but with much lower intensity. When the environment was adjusted to $100 \%$ oxygen, the PC-PCL fiber was barely visible in less than a second; the response times of these fibers are $\sim 0.1 \mathrm{~s}$. This observation follows the same trend as indicated by the spectra for other fiber mat measurements. The oxygen information carried by individual fibers provides the opportunity to obtain quantitative data from these images by intensity measurement and analysis. We have previously shown [9] that pixel intensities taken along individual fibers could potentially be used to map oxygen gradients. When the intensity contributed by the background was subtracted from the overall intensity attributed to the fiber, calibration curves can be obtained by measuring the average intensity of the fiber. Figure 3 demonstrates an example of calibration for the single fiber imaged at various gaseous oxygen concentrations. The intensity measured from the images was converted by the same Stern-Volmer equation used in the spectroscopy testing. The plot maintains the overall linearity with an adjusted $R^{2}$ of 0.9907 thanks to the well dispersion of the PdTFPP in PC fiber matrix. The ideal matrix material serves as solvent for the luminescent probe, provides appropriate mechanical support, improves selectivity by preventing penetrating of interference species such as iodides and, most importantly, adjusts the quenching behavior of the sensor. [28] 


\section{Biological Evaluation}

To evaluate the biocompatibility of the oxygen-sensitive core-shell fibers, dispersed U251 glioma cells were first cultured on the fibers for $48-72 \mathrm{~h}$. Incorporation of the probe could conceivably then lead to leaching of the porphyrin when the sensors are used in a liquid environment, affecting both sensor calibration and possibly causing cytotoxicity. However, Figure 4 shows that cells adhered to the fibers normally by elongating in the direction of fiber alignment. This suggests that aspects of the fiber chemistry did not interfere with either adhesion or morphology. These results agree with prior studies showing that nanofibers of similar composition to those containing the oxygen sensor had negligible effects on cell adhesion, motility and viability over periods as long as 48h [20]. We hypothesize that both the

protective PCL shell and the hydrophobic, water-resistant nature of PC core may have prevented any undesirable probe leaching. Individual dispersed cells cultured under normoxia or hypoxia and imaged similarly did not reveal oxygen gradients in their proximity i.e., the phosphorescence emission along the fibers was unchanged under the cells. We believe that this was due to limited oxygen consumption by individual cells and rapid oxygen diffusion from their surroundings that served to maintain homogeneous oxygen concentrations across the entire area.

\section{Microenvironmental Oxygen Gradients}

To further test our oxygen-sensor fibers with cell masses that are more representative of a micro-tumor (with a hypoxic core), we analyzed the phosphorescence of fibers in contact with spherical aggregates of U251 glioma cells (Figure 5). Aggregates were cultured in normoxic conditions before imaging ("non-primed aggregates") and the environment of the chamber was adjusted from normoxia to hypoxia $\left(1 \% \mathrm{O}_{2}\right)$ during the TIRF imaging session. Due to the large size of the aggregates, multiple images were taken in sequence and stitched together to show that cell fluorescence was unaffected by rapid changes in oxygen levels 
(Figure 5a-b) as the fibers increased in intensity from normoxia to hypoxia, as expected (Figure $5 c-d$ ). Analysis of the fibers under versus outside the aggregate failed to reveal a significant oxygen gradient between the aggregate mass and its surroundings (Figure 5e-f), suggesting an $\mathrm{O}_{2}$ intake by the aggregate actively compensated by diffusion from its surroundings.

However, when similar cell aggregates were first cultured under hypoxia $\left(1 \% \mathrm{O}_{2}\right)$ for 48h before imaging ("hypoxia-primed aggregates"), the TIRF images revealed quite different results (Figure 6). When imaged under environmental hypoxia $\left(1 \% \mathrm{O}_{2}\right)$ conditions, there was no difference in the median fiber phosphorescence intensity under versus outside the aggregate (Figure 6b, 6e). However, when the environment was gradually allowed to become normoxic (Figure 6c, 6f), the fibers under the aggregate retained a significant amount of phosphorescence showing that they continued to sense a low-oxygen condition (Figure $6 \mathrm{~g}$ ). We separately verified that these hypoxia-primed aggregates were indeed displaying hypoxiainduced features such as increased expression of HIF-1 $\alpha$ and the hypoxia-inducible growth factor VEGF (Figure 7a-b). The molecular mechanism by which hypoxia-primed aggregates exhibited "hypoxic footprints" versus non-primed aggregates (Figure 5) is unclear. Analysis of the OXPHOS components that reduce molecular $\mathrm{O}_{2}$ to $\mathrm{H}_{2} \mathrm{O}$ showed that oxidative phosphorylation was likely downregulated (Figure 7c), suggesting that the low $\mathrm{O}_{2}$ levels in hypoxic-primed aggregates exposed to normoxia was unlikely to be caused by increased mitochondrial consumption of $\mathrm{O}_{2}$ (see discussion below).

\section{Discussion}

This work shows progress towards using electrospun core-shell nanofibers as biocompatible, optical oxygen sensors for cell biology applications. We previously used this technology to show that scaffold modulus plays a critical role in differentially regulating the expression of Sox9 and Runx2 transcription factors in stem cells. [29]. However, the use of 
electrospun core-shell fibers as oxygen sensors provides additional practical advantages: a) easy manufacturing allowing adjustments of morphology and dimensions to alter sensor performance; b) excellent biocompatibility in direct biological applications; c) small fiber diameters and overall porous structure allowing rapid gas exchange rate and fast response and d) ease of miniaturization and integration with well-established devices such as bioreactors and cell culture plates.

In our recent paper [13], we combined this electrospun fiber-based platform with an oxygen-sensitive ruthenium compound to develop a fiber-based optical oxygen sensor. The sensor was shown to be capable of responding rapidly to changes in the oxygen content of the surrounding gaseous/aqueous environment. Using $0.5 \mu \mathrm{m}$ diameter electrospun polycaprolactone (PCL) fiber containing an oxygen-sensitive probe, tris (4,7- 22 diphenyl-1,10phenanthroline) ruthenium(II) dichloride, we observed a response time of $0.9 \pm 0.12 \mathrm{~s}$ while that for the corresponding film was more than two orders of magnitude slower. A more than 10-fold increase in PCL fiber diameter slightly reduced oxygen sensitivity while having only minor effects on response time.

In subsequent work [30], we reported the successful fabrication of polydimethylsiloxane (PDMS) fibers containing oxygen-sensitive probes by electrospinning and the applications of these fibers as optical oxygen sensors for both gaseous and dissolved oxygen. There, the protective PCL 'shell' maintained the fiber morphology during the slow curing of the liquid PDMS result while also increasing biocompatibility. This strategy allowed the dissolution tris(4,7-diphenyl-1,10-phenanthroline) ruthenium(II) $(\mathrm{Ru}(\mathrm{dpp}))$ and platinum octaethylporphyrin (PtOEP) into the PDMS core. All combinations exhibited excellent Stern-Volmer linearity due to the porous nature of the fibers and the excellent oxygen permeability of PDMS. We also established $\mathrm{Pd}(\mathrm{II})$ meso-tetra(pentafluorophenyl)porphine (PdTFPP) as having one of the highest oxygen-free intensities along with a rapid response ( 0.1s).[31, 32] Glioma cell lines (CNS1, U251) and glioma-derived primary cells (GBM34) were cultured on the fiber revealing 
negligible differences in biological behavior suggesting that the presence of the sensing molecules within the core carries with it no strong cytotoxic effects.

Polycarbonate fiber by itself is difficult to handle due to its relatively high modulus. Achieving small diameter electrospun fibers from PC is also problematic. Fortunately, the addition of a PCL 'shell' greatly improved these aspects. In this study, most samples were prepared by electrospinning of aligned fibers on glass coverslips and these coverslips were then integrated with a cell culture chamber compatible with microscopy imaging. Alternatively, fibers could be electrospun on polystyrene sheets and readily integrated with 24 or 96 well plates to produce standard cell culture plates with the ability to obtain oxygen information either using a plate reader or by direct imaging. Our previous publication [31] has shown that the surface and mechanical properties of the core-shell fibers can be independently adjusted for different biological applications. The excellent optical property of polycarbonate proved beneficial for current study in spite of the presence of the relatively translucent PCL as a shell present on the PC fiber surface. The signal output of these PC-PCL fibers is thus significantly higher than PCL fibers under the same excitation conditions. This allows the use of lower luminescent molecule concentrations that can still provide sufficient emission while reducing the risk of probe molecule aggregation.

In this study we show that core-shell PC-PCL fibers are similarly biocompatible and can be successfully used to report variations in oxygen content within cell cultures and even beneath tumor cell aggregates. We have shown that oxygen sensing is feasible for adherent cells that are chemically 'shielded' from the oxygen sensing porphyrin by the 'shell' of the nanofiber. This sensing approach combines the brightness and photostability advantages of probe-containing core with fast response and localization provided by the nanofiber host. Denser arrays of aligned fibers with small sensitive regions could allow 'mapping' of oxygen concentrations with unprecedented resolution. Moreover, two-photon laser scanning 
microscopy could be possibly used to map 3D oxygen distribution with submicron spatial resolution.

As the localized fluorescence of Fig. 2 establishes, the 'footprint' of the hypoxicconditioned U251 aggregate ( 250,000 cells) retains low oxygen concentrations $(\sim 1 \%)$ beneath it while the oxygen sensitive dye is quenched by the normoxia of its surroundings. When not cultured under hypoxia, no net differences in fluorescence under or around the agglomerates were observed. Hypoxic regions can be detected beneath tumor aggregates $\sim 500 \mu \mathrm{m}$ in size or approximately $0.64 \%$ of the mean recurrent tumor dimension of $78.6 \mathrm{~mm}$ or $17.86 \%$ of the smallest dimension $(2.8 \mathrm{~mm})$ detectable by MRI [33]. This relates to conditions observed in vivo in that hypoxia in brain cancer serves as an initiating factor [34-37] driving tumor cells to disperse throughout both the surrounding grey and white matter. GBM commonly spreads via migration along the aligned fiber found within white matter tracts, including the corpus callosum, resulting in tumor spread across both brain hemispheres ("butterfly pattern") characteristic of highly metastatic disease that has reached an incurable state [38, 39]. In vitro, when these glioma "neurospheres" are cultured on aligned fiber the cells detach and migrate in the fiber direction over distances 6 -fold greater than in the perpendicular direction [40].

These results establish that a specific goal of this study - the application of oxygensensing fibers to characterize the local oxygen microenvironment of brain tumor cells known to migrate in response to hypoxia $[41,42]$ - has been met. In the microenvironment of solid tumors, hypoxia regulates the progression to chemoresistance and metastasis [43] through gene expression and metabolic changes that make cells less dependent on oxygen to obtain energy [36, 44-46]. Embedded in tumors, sensors like these could help develop quantitative models of real-time oxygen distribution in and around tumor masses critical to understanding how local conditions can promote tumor malignancy or reduce the efficacy of chemotherapeutics. We hypothesize that denser nanofiber arrays having increased spatial 
resolution will improve detection of intra-aggregate oxygen gradients that were not observable in our normoxic cells likely due to immediate oxygen quenching of the sensor fibers. These arrays could be used to test the response of glioblastoma aggregates to chemotherapeutics and to identify drugs altering the use of $\mathrm{O}_{2}$ by tumor cell aggregates in hypoxia or normoxia.

Interestingly, although our fibers did not delineate oxygen gradients in individual tumor cells, they were sensitive enough to detect marked differences in oxygen levels across tumor aggregates pre-cultured in hypoxia for 48h. The reduced $\mathrm{O}_{2}$ levels in "hypoxia-primed" aggregates that returned to normoxia are likely caused by increased mitochondrial use of $\mathrm{O}_{2}$ as the OXPHOS components in those cells were downregulated (Figure 7) as expected in hypoxic cells [47]. A likely possibility is that "hypoxia-primed" aggregates converted $\mathrm{O}_{2}$ to superoxide radicals when they were returned to normoxia [3, 48-50], resulting in a decrease of available molecular oxygen visualized by the establishment of the hypoxic 'footprint' under the aggregates. Although the specific molecular mechanism by which hypoxic priming decreased available $\mathrm{O}_{2}$ in the tumor aggregates is beyond the scope of the present study, the results underscore the ability of our oxygen-sensing fibers to visualize oxygen gradients within microtumor masses.

\section{Conclusions}

In summary, this work suggests that polycarbonate-polycaprolactone fibers in the form of a core-shell fiber can successfully act as hosts allowing microscale oxygen sensing thanks to suitable gas permeability, optical properties, superior chemical stability, compatibility with the luminescent probe and appropriate surface properties needed for biological applications. By taking advantage of the high sensitivity and fast response of these sensors, we were able to locate and image hypoxic regions beneath cultured glioblastoma cell line aggregates, showing how they can be preserved in spite of surrounding areas of normoxia. The widely utilized electrospinning platform provides opportunities to construct ECM-like structures having 
both controlled morphology, chemistry and integrated oxygen sensing to lead to a better understanding of the how regions of stable hypoxia can develop and control tumor cell behavior. This chemically and physically flexible model allows analysis of glioma cell migration while recapitulating in vivo cell morphology, potentially allowing identification of localized oxygen conditions that control the efficacy of pharmacological inhibitors of invasion.

\section{Acknowledgments}

This work was partly supported by research grants from the National Science Foundation under Grant Nos. CBET- 1033991, EEC-0425626 and the Ohio State Pelotonia Foundation. Any opinions, findings, and conclusions or recommendations expressed in this material are those of the authors and do not necessarily reflect the views of the National Science Foundation or the National Institutes of Health. We also thank Carlos Castro for graciously allowing access to his total internal reflectance fluorescence microscope.

\section{References}

[1] Vaupel P, Briest S, Höckel M. Hypoxia in breast cancer: Pathogenesis, characterization and biological/therapeutic implications. Wiener Medizinische Wochenschrift. 2002;152:334-42.

[2] Vaupel P, Mayer A, Höckel M. Tumor Hypoxia and Malignant Progression. In: Chandan KS, Gregg LS, editors. Methods Enzymol: Academic Press; 2004. p. 335-54.

[3] Dewhirst MW, Cao Y, Moeller B. Cycling hypoxia and free radicals regulate angiogenesis and radiotherapy response. Nat Rev Cancer. 2008;8:425-37.

[4] Harrison L, Blackwell K. Hypoxia and anemia: Factors in decreased sensitivity to radiation therapy and chemotherapy? Oncologist. 2004;9:31-40.

[5] Gilkes DM, Semenza GL, Wirtz D. Hypoxia and the extracellular matrix: drivers of tumour metastasis. Nat Rev Cancer. 2014;14:430-9.

[6] Vaupel P, Hoeckel M, Mayer A. Detection and characterization of tumor hypoxia using $\mathrm{pO}(2)$ histography. Antioxidants \& Redox Signaling. 2007;9:1221-35.

[7] Clark LC. MONITOR AND CONTROL OF BLOOD AND TISSUE OXYGEN TENSIONS. Transactions American Society for Artificial Internal Organs. 1956;2:41-\&.

[8] Wang X-d, Wolfbeis OS. Optical methods for sensing and imaging oxygen: materials, spectroscopies and applications. Chem Soc Rev. 2014;43:3666-761.

[9] Ruipeng Xue GC, Kris Richardson, Andre Palmer, Mariano Viapiano, John J. Lannutti. Microscale Sensing of Oxygen via Encapsulated Porphyrin Nanofibers: Effect of Indicator and Polymer 'Core' Permeability. ACS Appl Mater Interfaces. 2015;7:8606-14. 
[10] Schreml S, Meier RJ, Wolfbeis OS, Maisch T, Szeimies R-M, Landthaler M, et al. 2D luminescence imaging of physiological wound oxygenation. Exp Dermatol. 2011;20:550-4.

[11] Dmitriev R, Papkovsky D. Optical probes and techniques for $\mathrm{O} 2$ measurement in live cells and tissue. Cell Mol Life Sci. 2012;69:2025-39.

[12] Cheema U, Rong Z, Kirresh O, MacRobert AJ, Vadgama P, Brown RA. Oxygen diffusion through collagen scaffolds at defined densities: implications for cell survival in tissue models. Journal of Tissue Engineering and Regenerative Medicine. 2012;6:77-84.

[13] Xue R, Behera P, Viapiano MS, Lannutti JJ. Rapid response oxygen-sensing nanofibers. Materials Science and Engineering: C. 2013;33:3450-7.

[14] Xue R, Behera P, Xu J, Viapiano MS, Lannutti JJ. Polydimethylsiloxane core-polycaprolactone shell nanofibers as biocompatible, real-time oxygen sensors. Sens Actuators, B. 2014;192:697-707.

[15] Ostrom QT, Gittleman H, Liao P, Rouse C, Chen Y, Dowling J, et al. CBTRUS statistical report: primary brain and central nervous system tumors diagnosed in the United States in 2007-2011. Neuro Oncol. 2014;16 Suppl 4:iv1-63.

[16] Wen PY, Kesari S. Malignant gliomas in adults. N Engl J Med. 2008;359:492-507.

[17] Keunen O, Johansson M, Oudin A, Sanzey M, Rahim SA, Fack F, et al. Anti-VEGF treatment reduces blood supply and increases tumor cell invasion in glioblastoma. Proc Natl Acad Sci U S A. 2011;108:374954.

[18] Barcellos-Hoff MH, Newcomb EW, Zagzag D, Narayana A. Therapeutic targets in malignant glioblastoma microenvironment. Seminars in radiation oncology. 2009;19:163-70.

[19] Viapiano MS, Lawler SE. Glioma invasion: Mechanisms and Therapeutic Challenges. In: Van Meir E, editor. CNS Cancer: Models, Prognostic Factors and Targets. New Jersey: Humana Press; 2009. p. 121952.

[20] Johnson J, Nowicki MO, Lee CH, Chiocca EA, Viapiano MS, Lawler SE, et al. Quantitative analysis of complex glioma cell migration on electrospun polycaprolactone using time-lapse microscopy. Tissue engineering Part C, Methods. 2009;15:531-40.

[21] Agudelo-Garcia PA, De Jesus JK, Williams SP, Nowicki MO, Chiocca EA, Liyanarachchi S, et al. Glioma cell migration on three-dimensional nanofiber scaffolds is regulated by substrate topography and abolished by inhibition of STAT3 signaling. Neoplasia (New York, NY). 2011;13:831-40.

[22] Lannutti J, Reneker D, Ma T, Tomasko D, Farson D. Electrospinning for tissue engineering scaffolds. Mater Sci Eng, C. 2007;27:504-9.

[23] Qu H, Wei S, Guo Z. Coaxial electrospun nanostructures and their applications. Journal of Materials Chemistry A. 2013;1:11513-28.

[24] Nam J, Huang Y, Agarwal S, Lannutti J. Materials selection and residual solvent retention in biodegradable electrospun fibers. J Appl Polym Sci. 2008;107:1547-54.

[25] Papkovsky DB, Ponomarev GV, Trettnak W, Oleary P. PHOSPHORESCENT COMPLEXES OF PORPHYRIN KETONES - OPTICAL-PROPERTIES AND APPLICATION TO OXYGEN SENSING. Anal Chem. 1995;67:4112-7.

[26] DeGraff BA, Demas JN. Luminescence-Based Oxygen Sensors Reviews in Fluorescence 2005. In: Geddes CD, Lakowicz JR, editors.: Springer US; 2005. p. 125-51.

[27] Papkovsky D, O'Riordan T. Emerging Applications of Phosphorescent Metalloporphyrins. Journal of Fluorescence. 2005;15:569-84.

[28] Klimant I, Wolfbeis OS. OXYGEN-SENSITIVE LUMINESCENT MATERIALS BASED ON SILICONE-SOLUBLE RUTHENIUM DIIMINE COMPLEXES. Anal Chem. 1995;67:3160-6.

[29] Nam J, Johnson JK, Lannutti JJ, Agarwal S. Modulation of Embryonic Mesenchymal Progenitor Cell Differentiation via Control over Pure Mechanical Modulus in Electrospun Nanofibers. Acta Biomaterialia. 2011;7:1516-24. 
[30] Xue R, Behera P, Viapiano MS, Lannutti JJ. Rapid response oxygen-sensing nanofibers. Materials Science \& Engineering C-Materials for Biological Applications. 2014;33:3450-7.

[31] Xue RP, Behera P, Xu JS, Viapiano MS, Lannutti JJ. Polydimethylsiloxane core-polycaprolactone shell nanofibers as biocompatible, real-time oxygen sensors. Sensors and Actuators B-Chemical. 2014;192:697-707.

[32] Xue R, Ge C, Richardson K, Palmer A, Viapiano M, Lannutti JJ. Microscale Sensing of Oxygen via Encapsulated Porphyrin Nanofibers: Effect of Indicator and Polymer "Core" Permeability. Acs Applied Materials \& Interfaces. 2015;7:8606-14.

[33] Dempsey MF, Condon BR, Hadley DM. Measurement of tumor "Size" in recurrent malignant glioma: 1D, 2D, or 3D? American Journal of Neuroradiology. 2005;26:770-6.

[34] Zhong H, De Marzo AM, Laughner E, Lim M, Hilton DA, Zagzag D, et al. Overexpression of hypoxiainducible factor 1 alpha in common human cancers and their metastases. Cancer Research. 1999;59:5830-5.

[35] Semenza GL. Hypoxia, clonal selection, and the role of HIF-1 in tumor progression. Critical Reviews in Biochemistry and Molecular Biology. 2000;35:71-103.

[36] Harris AL. Hypoxia - A key regulatory factor in tumour growth. Nature Reviews Cancer. 2002;2:38-47. [37] Tatum JL, Kelloff GJ, Gillies RJ, Arbeit JM, Brown JM, Chao KSC, et al. Hypoxia: Importance in tumor biology, noninvasive measurement by imaging, and value of its measurement in the management of cancer therapy. International Journal of Radiation Biology. 2006;82:699-757.

[38] Agrawal A. Butterfly glioma of the corpus callosum. Journal of Cancer Research and Therapeutics. 2009;5:43-5.

[39] Dziurzynski K, Blas-Boria D, Suki D, Cahill DP, Prabhu SS, Puduvalli V, et al. Butterfly glioblastomas: a retrospective review and qualitative assessment of outcomes. Journal of Neuro-Oncology. 2012;109:555-63.

[40] Johnson J, Nowicki MO, Lee CH, Chiocca EA, Viapiano MS, Lawler SE, et al. Quantitative Analysis of Complex Glioma Cell Migration on Electrospun Polycaprolactone Using Time-Lapse Microscopy. Tissue Engineering Part C-Methods. 2009;15:531-40.

[41] Cooper LA, Gutman DA, Chisolm C, Appin C, Kong J, Rong Y, et al. The tumor microenvironment strongly impacts master transcriptional regulators and gene expression class of glioblastoma. The American journal of pathology. 2012;180:2108-19.

[42] Rong Y, Durden DL, Van Meir EG, Brat DJ. 'Pseudopalisading' necrosis in glioblastoma: a familiar morphologic feature that links vascular pathology, hypoxia, and angiogenesis. JNeuropatholExpNeurol. 2006;65:529-39.

[43] Ruan K, Song G, Ouyang G. Role of Hypoxia in the Hallmarks of Human Cancer. J Cell Biochem. 2009;107:1053-62.

[44] Powis G, Kirkpatrick L. Hypoxia inducible factor-1 alpha as a cancer drug target. Molecular Cancer Therapeutics. 2004;3:647-54.

[45] Pouyssegur J, Dayan F, Mazure NM. Hypoxia signalling in cancer and approaches to enforce tumour regression. Nature. 2006;441:437-43.

[46] Maxwell PH, Pugh CW, Ratcliffe PJ. Activation of the HIF pathway in cancer. Current Opinion in Genetics \& Development. 2001;11:293-9.

[47] Solaini G, Baracca A, Lenaz G, Sgarbi G. Hypoxia and mitochondrial oxidative metabolism. Biochimica et biophysica acta. 2010;1797:1171-7.

[48] Hsieh CH, Lee CH, Liang JA, Yu CY, Shyu WC. Cycling hypoxia increases U87 glioma cell radioresistance via ROS induced higher and long-term HIF-1 signal transduction activity. Oncology reports. 2010;24:1629-36.

[49] Griguer CE, Oliva CR, Kelley EE, Giles GI, Lancaster JR, Jr., Gillespie GY. Xanthine oxidase-dependent regulation of hypoxia-inducible factor in cancer cells. Cancer Res. 2006;66:2257-63. 
[50] Jeong JI, Lee YW, Kim YK. Chemical hypoxia-induced cell death in human glioma cells: role of reactive oxygen species, ATP depletion, mitochondrial damage and Ca2+. Neurochemical research. 2003;28:1201-11. 


\section{Figure Legends:}

Figure 1. Morphology (left), spectral sensitivity (middle), and imaging sensitivity (right) of the PC-PCL core-shell fibers containing PdTFPP fibers.

Figure 2. Reversibility (left) and calibration (right) in $37^{\circ} \mathrm{C}$ water

Figure 3. Single fiber sensing; the calibration plot obtained by measuring average intensity from the images of PC(PdTFPP) fiber at different oxygen concentrations (upper images) and the Stern-Volmer plot (lower image) obtained by measuring the average intensity from the image after background subtraction.

Figure 4. Oxygen-sensor fibers are biocompatible with glioma cells. A) Representative images of U251 glioblastoma cells (green) adhered to fibers in hypoxia $\left(1 \% \mathrm{O}_{2}\right)$ or normoxic $\left(21 \% \mathrm{O}_{2}\right)$ conditions. Cell adhesion was unaffected by the chemical make-up of the fibers. $\left.\mathrm{B}\right)$ Calibration curve for oxygen-sensing fibers in presence of individual cells. No differences in phosphorescence were detected between fiber segments in direct contact with individual cells versus distant, cell-free segments.

Figure 5. Oxygen-sensor fibers can measure environmental $\mathrm{O}_{2}$ in presence of cell aggregates. A-B) Representative images of a green fluorescent U251 cell aggregate cultured on top of oxygen sensing-fibers in hypoxic (A) or normoxic (B) conditions. C-D) Spatial map of phosphorescence intensity along the fibers (false-color scale) in hypoxic (C) or normoxic (D) conditions. Notice a particularly bright fiber crossing the image horizontally above the aggregate (possible outlier). The aggregate area was been delineated manually. E) 
Distribution of pixel intensity for fiber segments under the aggregate (" $\boldsymbol{A}$ ") or in the periphery outside the aggregate ("P'). The A/P ratios suggest similar median phosphorescence value for fibers under or outside the aggregate

Figure 6. Oxygen-sensor fibers can detect an oxygen gradient for hypoxia-primed aggregates. A) Brightfield image of a single, hypoxia-primed aggregate adhered to oxygensensor fibers. B-C) Phosphorescence of the corresponding fibers in hypoxia (B) and normoxia (C). D) Merged image of the aggregate in (A) and the subjacent fibers in (C). E-F) Spatial maps of phosphorescence intensity along the fibers (false-color scale) in hypoxic (E) or normoxic (F) conditions. G) Frequency distribution of pixel intensity under and outside the aggregate (as shown in Figure 5) The A/P ratios suggest that fibers under the aggregate keep sensing hypoxic conditions when the culture is returned to normoxia, therefore revealing a gradient from the core to the periphery of the aggregate.

Figure 7. Validation of hypoxic status for glioblastoma aggregates. "Hypoxia-primed" aggregates similar to those imaged in Figure 6 were cultured in hypoxia for $48 \mathrm{~h}$ and processed for Western blotting or ELISA. A) Increased expression of soluble VEGF in the medium of hypoxic versus normoxic aggregates ( ${ }^{* * *} p<0.001$ by Student's t-test). B) Increased expression of HIF-1a protein in hypoxic aggregates. C) Differential expression of four components of the OXPHOS mitochondrial cascade in hypoxic aggregates compared to normoxia. Results suggest minor or no changes in complex II and V and considerable downregulation in complex III and IV. 
Figure 1:
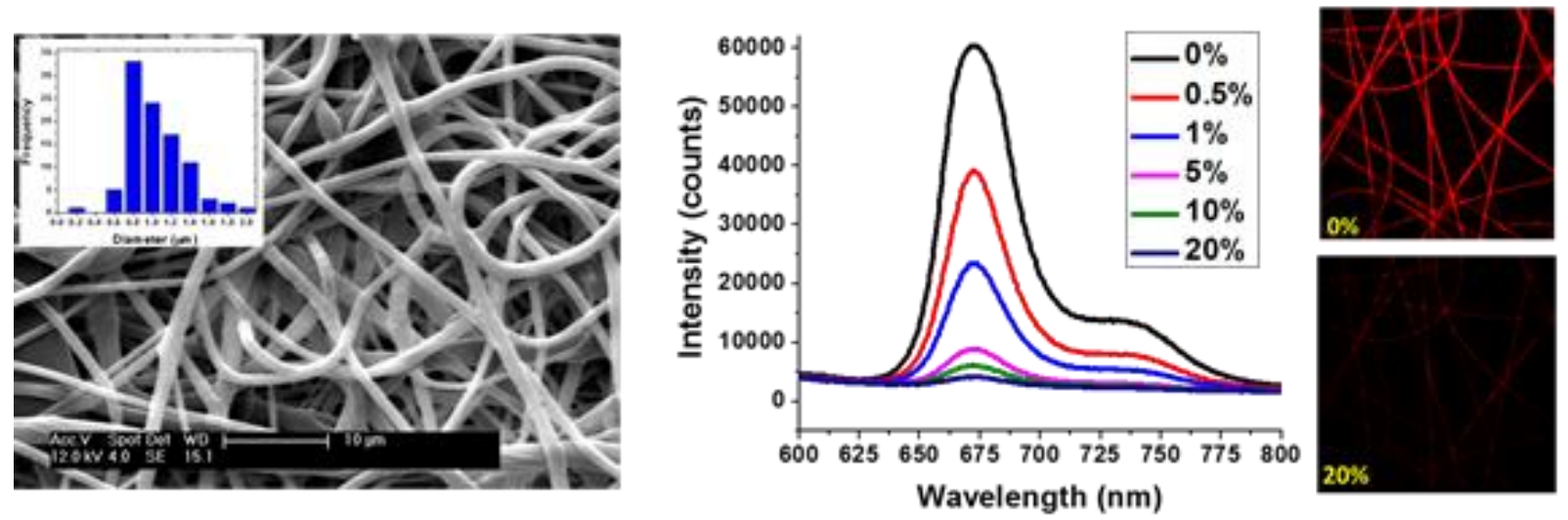

Figure 2:
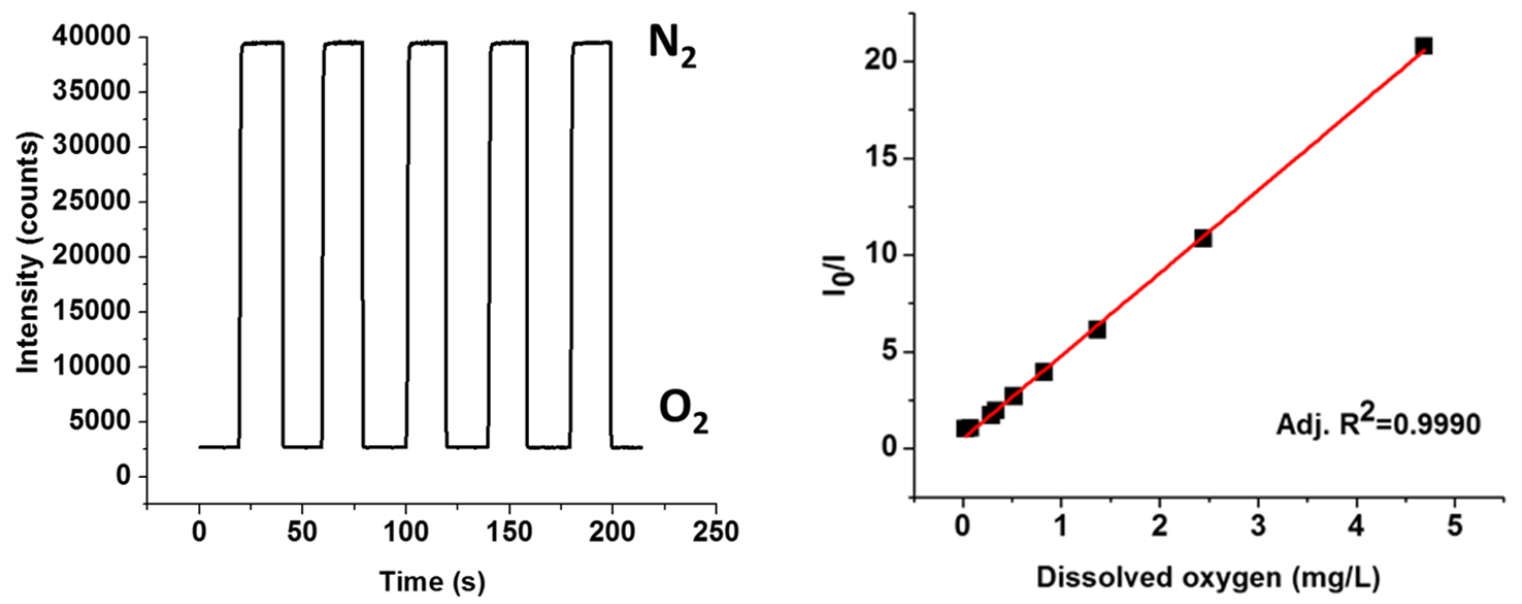
Figure 3:
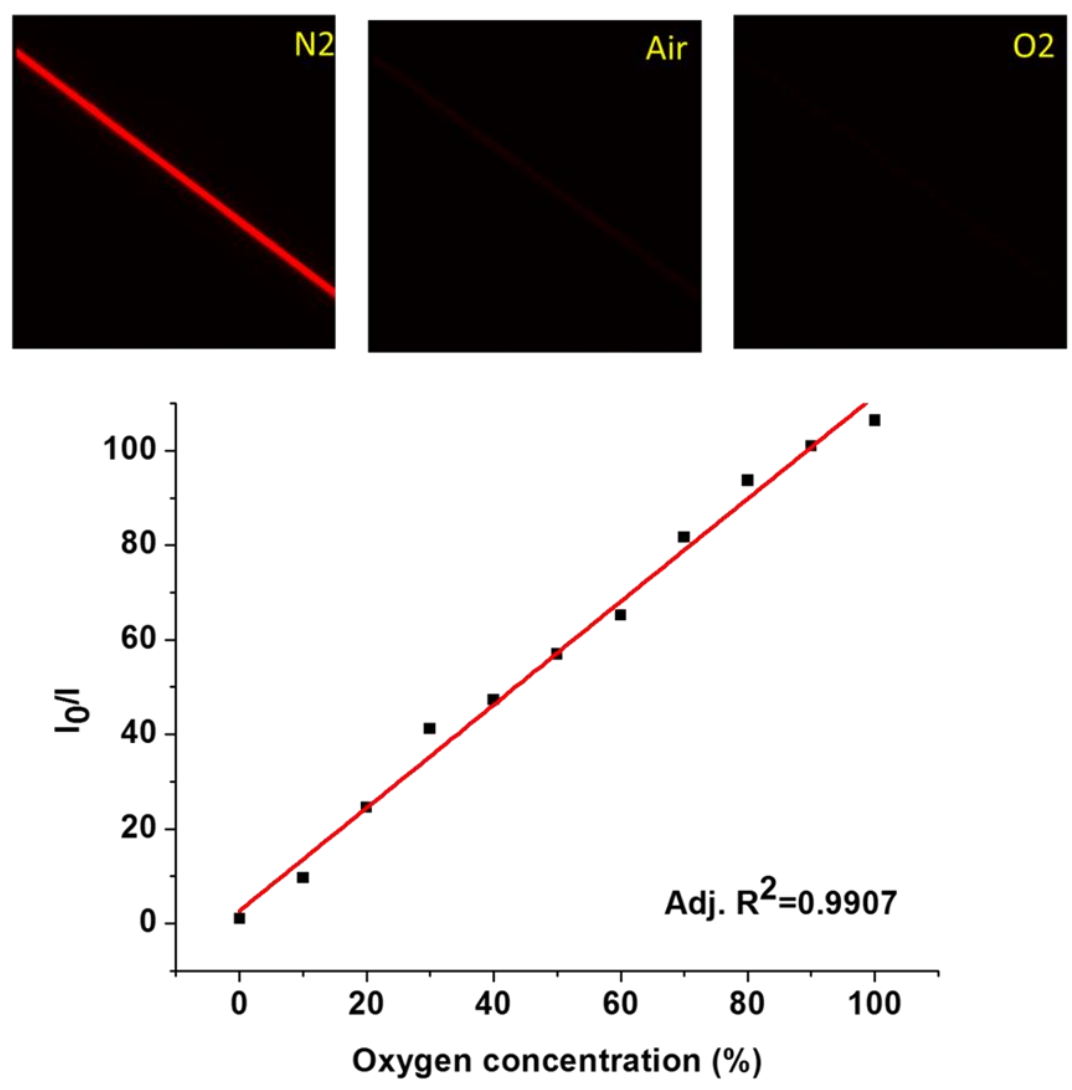

Figure 4:
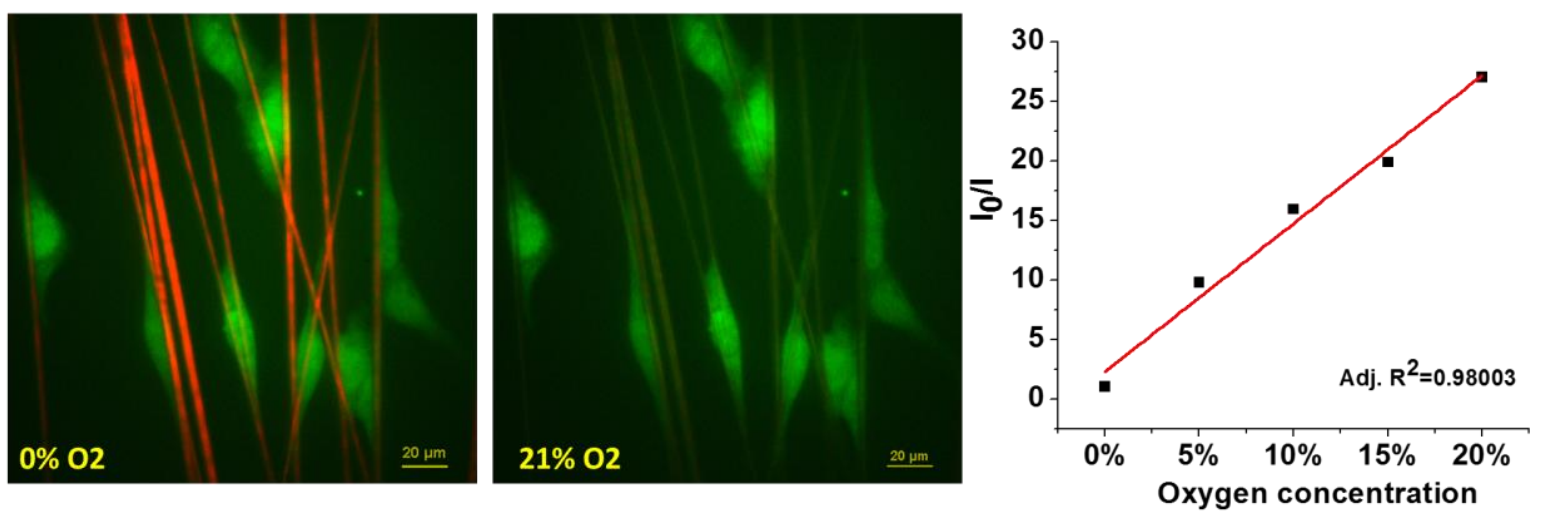
Figure 5:
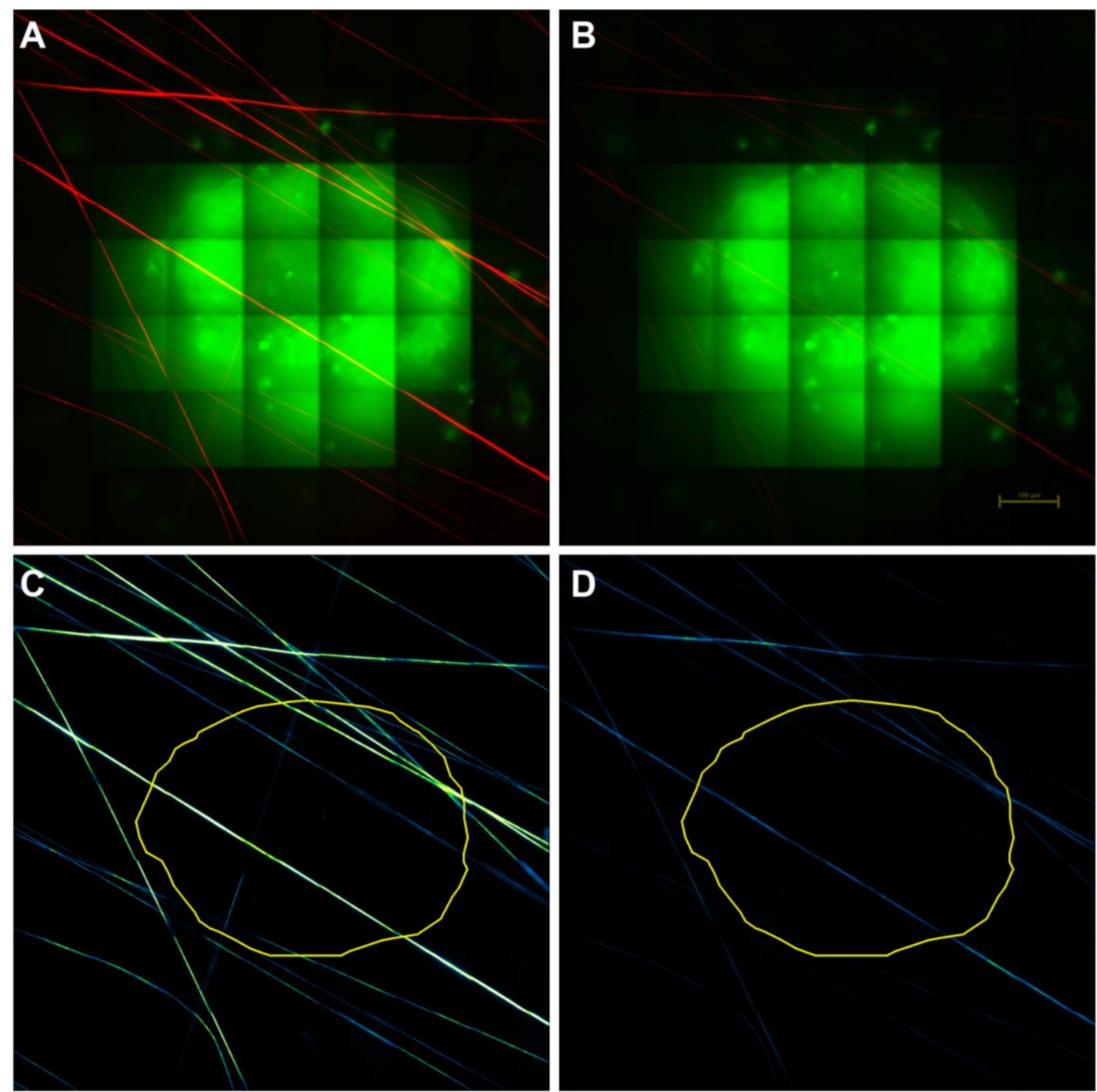

E

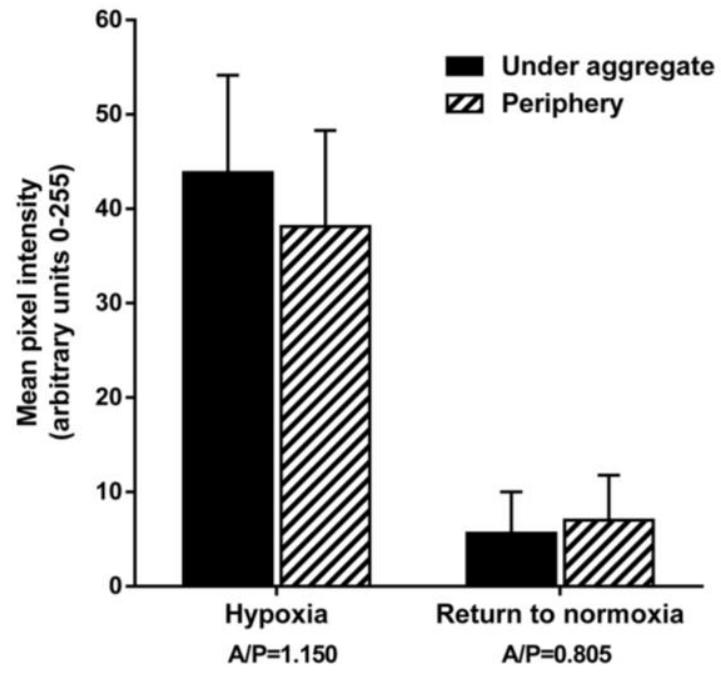


Figure 6:
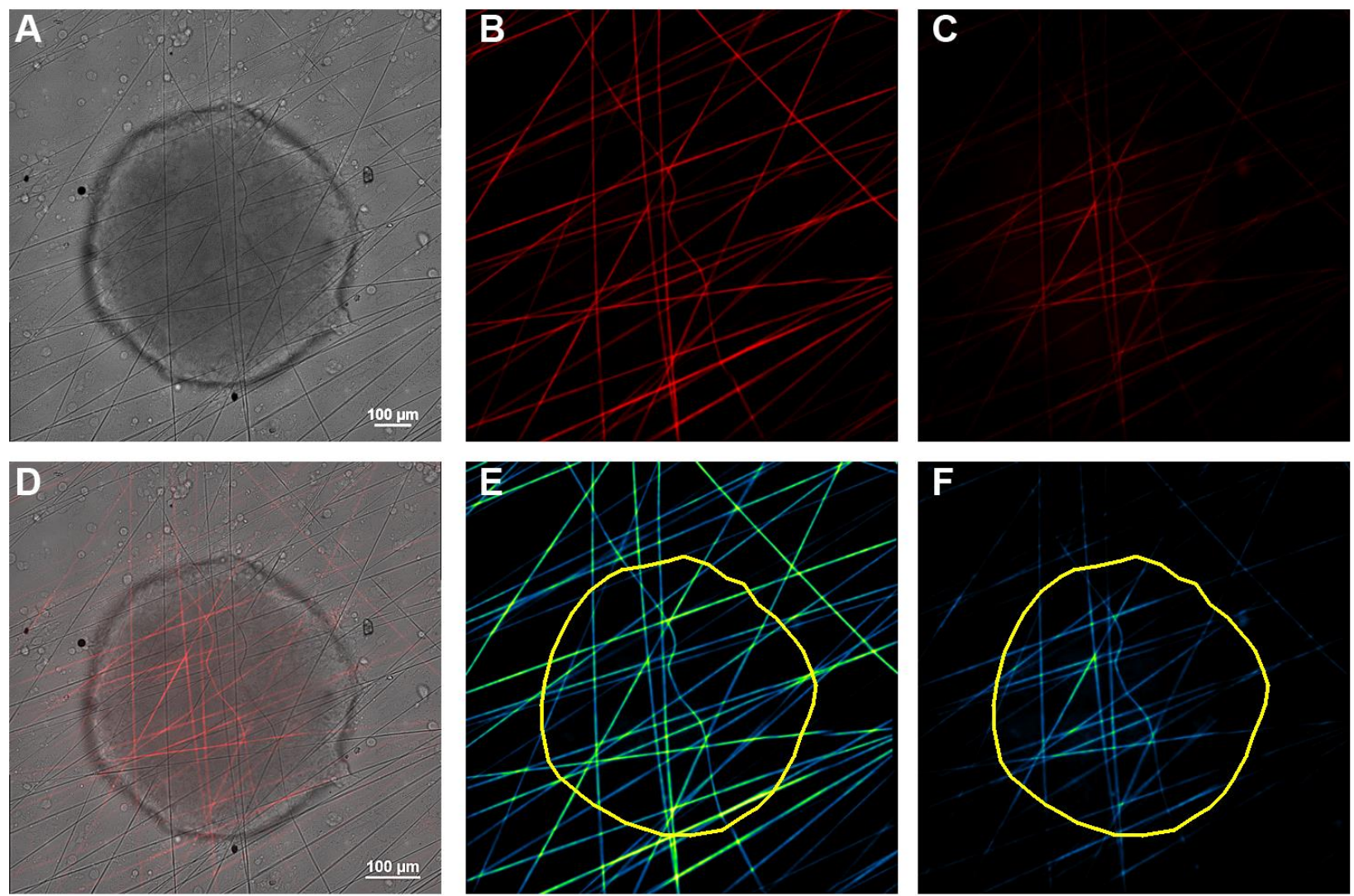

G

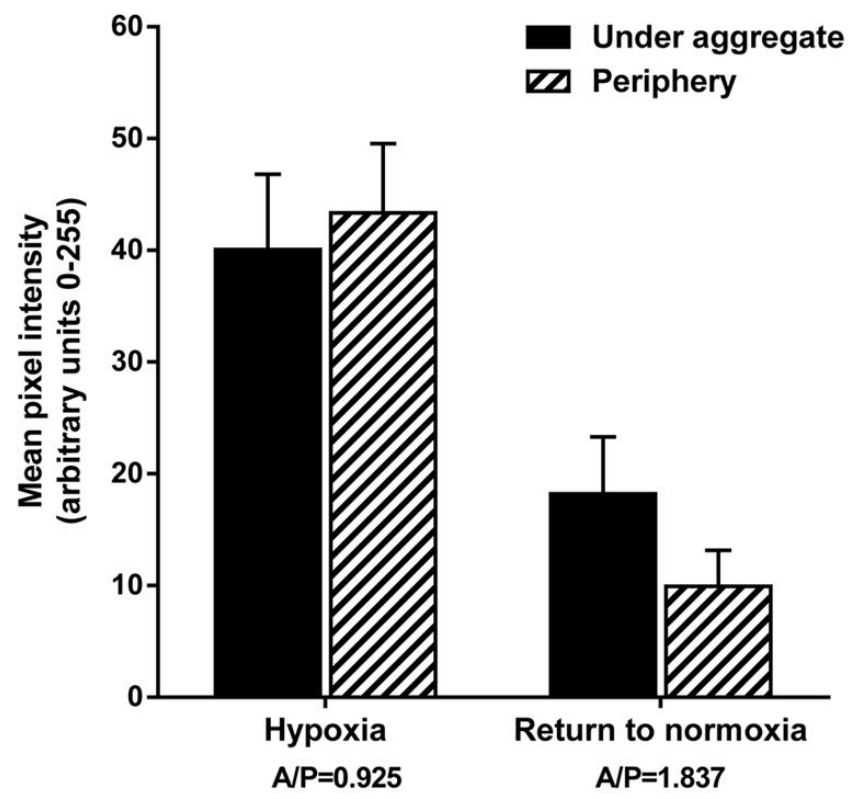


Figure 7:
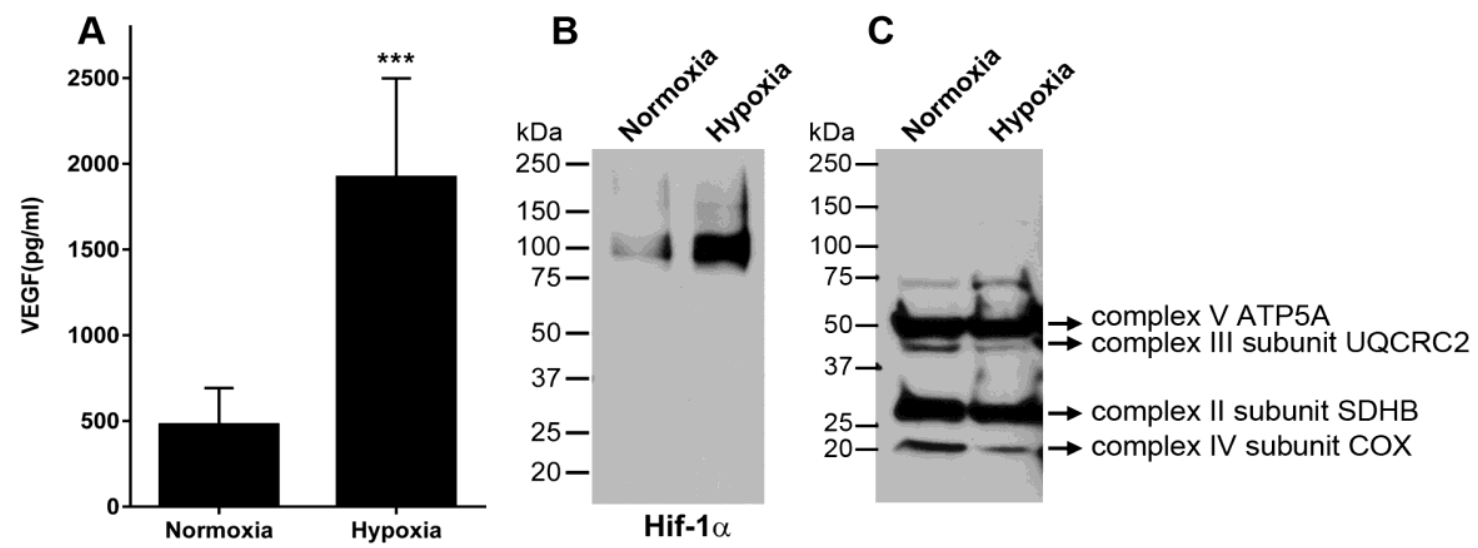